\title{
Special issue on impulsivity and compulsivity
}

\author{
TW Robbins $\cdot$ HV Curran $\cdot$ H de Wit
}

Received: 8 November 2011 / Accepted: 8 November 2011 / Published online: 29 November 2011

(C) Springer-Verlag 2011

This 'Special issue on impulsivity and compulsivity' revisits a theme first explored by Psychopharmacology in 1999 (146, 339-491). That issue of the journal focused on impulsivity and represented somewhat of a landmark for this topic in Psychopharmacology and perhaps for the field in general. It contained several now highly cited papers by several notable researchers in the field, including two seminal theoretical reviews. One was by John Evenden ('Varieties of impulsivity' Evenden 1999) and has been cited 481 times at last count on the ISI Web of Knowledge. The other, on impulsivity, frontostriatal dysfunction and drug abuse, was by David Jentsch and Jane Taylor (1999) and has been cited no fewer than 555 times.

As Evenden's review presciently anticipated, impulsivity can be defined in several ways, reflecting what may be a multifaceted construct, engaging several distinct neural systems (Dalley et al. 2011). Most definitions emphasise

\section{T. Robbins $(\square)$}

Behavioural and Clinical Neuroscience Institute and

Department of Experimental Psychology,

University of Cambridge,

Cambridge, UK

e-mail: twr2@cam.ac.uk

\section{H. Curran}

Clinical Psychopharmacology Unit,

Research Department of Clinical,

Health and Educational Psychology,

University College London,

Gower St., London WC1E 6BT, UK

e-mail: v.curran@ucl.ac.uk

\section{H. de Wit}

Human Behavioral Pharmacology Laboratory,

University of Chicago,

5841 S. Maryland Avenue, 60637, Chicago, IL, USA

e-mail: hdew@uchicago.edu the tendency towards maladaptive premature behaviour, the loss of motor or response inhibition, the performance of 'automatic urges or impulses', the tendency to avoid delay and rapidly discount the value of delayed rewards ('impulsive choice') and the lack of reflection in making decisions, often of a highly 'risky' type. In general, advances in understanding of impulsivity have stemmed from advances in its measurement in both humans and experimental animals, which may often proceed in parallel, using analogous tests such as the temporal discounting of reward or stop signal inhibition. Many of these methods are represented in this special issue. Roige et al. provide a detailed and timely review of the considerable and exciting recent work in mice (including genetic strains) on the same five-choice serial reaction attentional task used by Dalley et al. to measure impulsivity in rats.

Impulsivity refers to a constellation of traits that may be found in several neuropsychiatric disorders, including attention deficit/hyperactivity disorder (ADHD), substance abuse including alcoholism and cigarette smoking, mania, antisocial behaviour, as well as pathological aggression and depression (i.e. suicide). There have been suggestions that forms of impulsivity can be endophenotypes or intermediate phenotypes for some disorders. For example, rats that exhibit premature behaviour in the five-choice task appear to have a propensity for compulsive cocaine-seeking behaviour, even before they are actually exposed to the drug (Dalley et al. 2011). It is also possible that some abused drugs themselves may also cause impulsive behaviours (Perry and Carroll 2008). Impulsive behaviour may also be especially important in the relapse to drug seeking, as they may share overlapping neural mechanisms (see in this issue the study by Verdejo-Garcia et al.). Several forms of impulsivity are also prominent in ADHD, which often responds to treatment with the indirect catecholamine 
agonist methylphenidate. Several of the papers (e.g. by Winstanley et al., E. Robinson, Pattij et al., Fernando et al., Baarendse and Vanderschuren, and Kim et al.) in this special issue report effects of the catecholamine modulation of several forms of impulsive behaviour, with a special focus on noradrenergic effects. Serotonin has also been classically linked with some forms of impulsivity, and this hypothesis is explored in novel ways by Robinson et al., Crockett et al. and Blasio et al. in studies involving either humans or experimental animals. Other articles link research with rodents on impulsive behaviour to drug addiction models, in the tradition of Perry and Carroll (2008; e.g. Semenova et al., Schippers et al. and Kolokotroni et al.). This is mirrored by work with humans on substance abuse [e.g. on alcohol (Christiansen et al., Papachristou et al., Murphy and MacKillop) and nicotine (Ashare et al., Mitchell and Wilson, Addicott et al.) and also compulsive gambling (e.g. Andrade and Petry)]. Entirely faithful to the theme of this special issue is the review by Leeman and Potenza on compulsive gambling in humans.

By contrast, the construct of compulsivity has been less well-defined and investigated. Only a few researchers have examined the commonalities and differences between impulsive and compulsive behaviour (Hollander and Rosen 2000). For example, both constructs invoke the concept of top-down inhibitory control over behaviour. However, they appear to be most different in terms of their persistence. Compulsive behaviour is repetitive or perseverative in nature, despite adverse consequences. It is important to realize that such a criterion may apply to a single simple unconditioned response such as head movement (as in stereotyped behaviour induced by dopamine agonists) or more complex sequences of motor responses. Compulsive behaviour may often occur without reference to the original goal of such responding, and in this sense resembles habitlike behaviour, i.e. based on stimulus-response representations; the article by de Wit et al. pursues the implications of this concept using a dietary method to deplete central dopamine. Compulsivity is also readily measured by perseverative tendencies in reversal learning, a paradigm that is comprehensively reviewed by Izquierdo and Jentsch from both the animal and human perspective. Another intriguing animal model of compulsivity is scheduleinduced polydipsia (review by Moreno and Flores), which is of considerable interest in view of its excessive nature and individual variation, and which may be relevant not only to obsessive-compulsive disorder, the prototypical disorder of compulsive behaviour, but also possibly alcohol abuse. Resistance to extinction and narrowed attentional focus may also be features of compulsive behaviour not represented here. Given apparent differences in the various paradigms used to measure compulsivity, future research may well reveal different forms of compulsivity, just as there are different forms of impulsivity. Finally, FernandezSerrano et al. consider the proposition that cocaine use may entail compulsive, as well as impulsive, behavioural features, in parallel with some animal studies.

The large number of articles submitted to this special issue, as well as its ultimate size, is a testament to the vibrancy of this field. The notions of impulsivity and compulsivity are especially important in the clinical sphere as they point towards a more dimensional, as opposed to categorical, approach to the description of neuropsychiatric disorders ('impulsive-compulsive disorders'), with enormous implications for methods of diagnosis. It will be of great interest to see how the concepts of impulsivity and compulsivity evolve over the next decade and whether they retain their utility. The breadth of coverage in this issue suggests that they may have to be further dissected and refined as our understanding grows.

\section{References}

Dalley JW, Everitt BJ, Robbins TW (2011) Impulsivity, compulsivity, and top-down cognitive control. Neuron 69:680-694

Evenden JL (1999) Varieties of impulsivity. Psychopharmacology 146:348-361

Hollander E, Rosen J (2000) Impulsivity. J Psychopharmacol 14:S39-S44

Jenstch JD, Taylor JR (1999) Impulsivity resulting from frontostriatal dysfunction in drug abuse: implications for the control of behavior by reward-related stimuli. Psychopharmacology 146:373-390

Perry JL, Carroll ME (2008) The role of impulsive behavior in drug abuse. Psychopharmacology 200:1-26 